\title{
Multiple positive solutions for nonlocal boundary value problems of singular fractional differential equations
}

Xingqiu Zhang ${ }^{1,2}$ and Qiuyan Zhong ${ }^{1 *}$

${ }^{\text {"Correspondence: }}$

zhqy197308@163.com

'Department of Information

Engineering, Jining Medical College,

Jining, Shandong 272067, P.R. China

Full list of author information is

available at the end of the article

\begin{abstract}
We consider the existence of multiple positive solutions for the following nonlinear fractional differential equations of nonlocal boundary value problems:

$$
\left\{\begin{array}{l}
D_{0+}^{\alpha} u(t)+f(t, u(t))=0, \quad 0<t<1 \\
u(0)=0, \quad D_{0+}^{\beta} u(0)=0, \quad D_{0+}^{\beta} u(1)=\sum_{i=1}^{\infty} \xi_{i} D_{0+}^{\beta} u\left(\eta_{i}\right)
\end{array}\right.
$$

where $2<\alpha \leq 3,1 \leq \beta \leq 2, \alpha-\beta \geq 1,0<\xi_{i}, \eta_{i}<1$ with $\sum_{i=1}^{\infty} \xi_{i} \eta_{i}^{\alpha-\beta-1}<1$. Existence result of at least two positive solutions is given via fixed point theorem on cones. The nonlinearity $f$ may be singular both on the time and the space variables.
\end{abstract}

MSC: 26A33; 34B15; 34B18

Keywords: fractional differential equations; nonlocal boundary value problem; singularity; multiple positive solutions

\section{Introduction}

The purpose of this paper is to investigate the multiplicity of positive solutions for the following nonlocal boundary value problems of singular fractional differential equations:

$$
\left\{\begin{array}{l}
D_{0+}^{\alpha} u(t)+f(t, u(t))=0, \quad 0<t<1, \\
u(0)=0, \quad D_{0+}^{\beta} u(0)=0, \quad D_{0+}^{\beta} u(1)=\sum_{i=1}^{\infty} \xi_{i} D_{0+}^{\beta} u\left(\eta_{i}\right),
\end{array}\right.
$$

where $2<\alpha \leq 3,1 \leq \beta \leq 2, \alpha-\beta \geq 1,0<\xi_{i}, \eta_{i}<1$ with $\sum_{i=1}^{\infty} \xi_{i} \eta_{i}^{\alpha-\beta-1}<1, f \in C(J \times$ $\left.\mathbb{R}_{++}, \mathbb{R}_{+}\right), J=(0,1), \mathbb{R}_{+}=[0,+\infty), \mathbb{R}_{++}=(0,+\infty), D_{0+}^{\alpha}$ is the standard Riemann-Liouville's fractional derivative of order $\alpha$. The nonlinearity $f$ permits singularities at $t=0,1$ and $u=0$. A function $u \in C[0,1]$ is said to be a positive solution of BVP $(1)$ if $u(t)>0$ on $(0,1)$ and $u$ satisfies $(1)$ on $[0,1]$.

Recently, much attention has been paid on the study of nonlocal boundary value problems of fractional differential equations; see [1-16] and [17-25]. By virtue of the contraction map principle and the fixed point index theory, Bai [1] investigated the existence and uniqueness of positive solutions for the following fractional differential equation:

$$
D_{0+}^{\alpha} u(t)+f(t, u(t))=0, \quad 0<t<1,1<\alpha \leq 2
$$

(c) 2016 Zhang and Zhong. This article is distributed under the terms of the Creative Commons Attribution 4.0 International License (http://creativecommons.org/licenses/by/4.0/), which permits unrestricted use, distribution, and reproduction in any medium, provided you give appropriate credit to the original author(s) and the source, provide a link to the Creative Commons license, and indicate if changes were made. 
subject to three point boundary value conditions

$$
u(0)=0, u(1)=\mu u(\xi),
$$

where $0<\mu \xi^{\alpha-1}<1,0 \leq \mu \leq 1,0<\xi<1, f$ is continuous on $[0,1] \times[0,+\infty)$. When $f:[0,1] \times[0,+\infty) \rightarrow[0,+\infty)$ satisfies Carathéodory type conditions, by using some fixed point theorems, like Leray-Schauder nonlinear alternative and a mixed monotone method, Li et al. [2], Xu et al. [3, 4] obtained the existence and multiplicity results of positive solutions for the fractional differential equation (A) with fractional derivative in boundary conditions

$$
u(0)=0, \quad D_{0+}^{\beta} u(1)=\mu D_{0+}^{\beta} u(\xi), \quad 0 \leq \beta \leq 1 .
$$

In 2011, Lv [5] and Yang et al. [6] discussed the existence of minimal and maximal and the uniqueness of positive solutions for fractional differential equation (A) under multi-point boundary value conditions,

$$
u(0)=0, \quad D_{0+}^{\beta} u(1)=\sum_{i=1}^{m-2} \xi_{i} D_{0+}^{\beta} u\left(\eta_{i}\right), \quad 0 \leq \beta \leq 1
$$

Motivated by the above papers, when $2<\alpha \leq 3$ and $f$ is continuous, Li et al. [7] obtained the existence results of at least one and unique solutions for fractional differential equation (A) subject to more general multi-point boundary value conditions

$$
u(0)=0, \quad D_{0+}^{\beta} u(0)=0, \quad D_{0+}^{\beta} u(1)=\sum_{i=1}^{m-2} \xi_{i} D_{0+}^{\beta} u\left(\eta_{i}\right) .
$$

The tools to obtain the main results are the nonlinear alternative of the Leray-Schauder and the Banach contraction mapping principle.

Compared with the existing literature, this paper has the following two new features. First, different from [7], infinite-point boundary value conditions are considered in this paper. At the same time, the nonlinearity $f$ in this paper permits singularities with respect to both the time and the space variables which is seldom considered at present. Second, the purpose of this paper is to investigate the existence of multiple positive solutions for BVP (1). As to multiple positive solutions, it is worth pointing out that conditions imposed on $f$ are different from that in [4]. To achieve this goal, first we convert the expression of the unique solution into an integral form and then get the Green function BVP (1). After further discussion of the properties of the Green function, a suitable cone is constructed to obtain the main result in this paper by means of the Guo-Krasnoselskii fixed point theorem.

\section{Preliminaries and several lemmas}

Definitions and useful lemmas from fractional calculus theory can be found in the recent literature [26-28], we omit them here. 
Lemma 1 ([1]) Assume that $u \in C(0,1) \cap L(0,1)$ with a fractional derivative of order $\alpha>0$ that belongs to $C(0,1) \cap L(0,1)$. Then

$$
I_{0+}^{\alpha} D_{0+}^{\alpha} u(t)=u(t)+C_{1} t^{\alpha-1}+C_{2} t^{\alpha-2}+\cdots+C_{N} t^{\alpha-N}
$$

for some $C_{i} \in R, i=1,2, \ldots, N$, where $N$ is the smallest integer greater than or equal to $\alpha$.

Lemma 2 Let $y \in L^{1}[0,1]$ and $2<\alpha \leq 3$, the unique solution of

$$
\left\{\begin{array}{l}
D_{0+}^{\alpha} u(t)+y(t)=0, \\
u(0)=0, \quad D_{0+}^{\beta} u(0)=0, \quad D_{0+}^{\beta} u(1)=\sum_{i=1}^{\infty} \xi_{i} D_{0+}^{\beta} u\left(\eta_{i}\right),
\end{array}\right.
$$

is

$$
u(t)=\int_{0}^{1} G(t, s) y(s) \mathrm{d} s
$$

where

$$
G(t, s)=\frac{1}{p(0) \Gamma(\alpha)} \begin{cases}p(s)(1-s)^{\alpha-\beta-1} t^{\alpha-1}-p(0)(t-s)^{\alpha-1}, & 0 \leq s \leq t \leq 1, \\ p(s)(1-s)^{\alpha-\beta-1} t^{\alpha-1}, & 0 \leq t \leq s \leq 1,\end{cases}
$$

here $p(s)=1-\sum_{s \leq \eta_{i}} \xi_{i}\left(\frac{\eta_{i}-s}{1-s}\right)^{\alpha-\beta-1}$.

Proof By Lemma 1, we get

$$
u(t)=-I_{0^{+}}^{\alpha} y(t)+C_{1} t^{\alpha-1}+C_{2} t^{\alpha-2}+C_{3} t^{\alpha-3} .
$$

It follows from the condition $u(0)=0$ that $C_{3}=0$. Considering the relation $D_{0^{+}}^{\alpha} t^{\gamma}=$ $\frac{\Gamma(\gamma+1)}{\Gamma(\gamma-\alpha+1)} t^{\gamma-\alpha}$, we have

$$
\begin{aligned}
D_{0^{+}}^{\beta} u(t)= & D_{0^{+}}^{\beta}\left(-\int_{0}^{t} \frac{1}{\Gamma(\alpha)}(t-s)^{\alpha-1} y(s) \mathrm{d} s\right) \\
& +C_{1} \frac{\Gamma(\alpha)}{\Gamma(\alpha-\beta)} t^{\alpha-\beta-1}+C_{2} \frac{\Gamma(\alpha-1)}{\Gamma(\alpha-\beta-1)} t^{\alpha-\beta-2} .
\end{aligned}
$$

Since $2<\alpha \leq 3,1 \leq \beta \leq 2$, and $\alpha \geq \beta+1$, we have $-1 \leq \alpha-\beta-2 \leq 0$. Thus, $C_{2}=0$. As deduced by the boundary value condition $D_{0+}^{\beta} u(1)=\sum_{i=1}^{\infty} \xi_{i} D_{0+}^{\beta} u\left(\eta_{i}\right)$, we have

$$
\begin{aligned}
- & \frac{1}{\Gamma(\alpha-\beta)} \int_{0}^{1}(1-s)^{\alpha-\beta-1} y(s) \mathrm{d} s+C_{1} \frac{\Gamma(\alpha)}{\Gamma(\alpha-\beta)} \\
& =-\sum_{i=1}^{\infty} \frac{1}{\Gamma(\alpha-\beta)} \xi_{i} \int_{0}^{\eta_{i}}\left(\eta_{i}-s\right)^{\alpha-\beta-1} y(s) \mathrm{d} s+C_{1} \sum_{i=1}^{\infty} \xi_{i} \frac{\Gamma(\alpha)}{\Gamma(\alpha-\beta)} \eta_{i}^{\alpha-\beta-1},
\end{aligned}
$$

which implies that

$$
\begin{aligned}
C_{1} & =\int_{0}^{1} \frac{(1-s)^{\alpha-\beta-1}}{\Gamma(\alpha)\left(1-\sum_{i=1}^{\infty} \xi_{i} \eta_{i}^{\alpha-\beta-1}\right)} y(s) \mathrm{d} s-\sum_{i=1}^{\infty} \xi_{i} \int_{0}^{\eta_{i}} \frac{\left(\eta_{i}-s\right)^{\alpha-\beta-1}}{\Gamma(\alpha)\left(1-\sum_{i=1}^{\infty} \xi_{i} \eta_{i}^{\alpha-\beta-1}\right)} y(s) \mathrm{d} s \\
& =\int_{0}^{1} \frac{(1-s)^{\alpha-\beta-1} p(s)}{\Gamma(\alpha) p(0)} y(s) \mathrm{d} s
\end{aligned}
$$


where $p(s)=1-\sum_{s \leq \eta_{i}} \xi_{i}\left(\frac{\eta_{i}-s}{1-s}\right)^{\alpha-\beta-1}$. Thus, we have

$$
\begin{aligned}
u(t)= & -\int_{0}^{t} \frac{1}{\Gamma(\alpha)}(t-s)^{\alpha-1} y(s) \mathrm{d} s+\int_{0}^{1} \frac{(1-s)^{\alpha-\beta-1} p(s)}{\Gamma(\alpha) p(0)} y(s) \mathrm{d} s \cdot t^{\alpha-1} \\
= & \int_{0}^{t} \frac{p(s)(1-s)^{\alpha-\beta-1} t^{\alpha-1}-p(0)(t-s)^{\alpha-1}}{\Gamma(\alpha) p(0)} y(s) \mathrm{d} s \\
& +\int_{t}^{1} \frac{p(s)(1-s)^{\alpha-\beta-1} t^{\alpha-1}}{\Gamma(\alpha) p(0)} y(s) \mathrm{d} s \\
= & \int_{0}^{1} G(t, s) \mathrm{d} s .
\end{aligned}
$$

Lemma 3 The function $p(s)>0, s \in[0,1]$, and $p$ is nondecreasing on $[0,1]$.

Proof By direct computation, we have

$$
\begin{aligned}
p^{\prime}(s)= & \sum_{s \leq \eta_{i}} \xi_{i}(\alpha-\beta-1)\left(\eta_{i}-s\right)^{\alpha-\beta-2}(1-s)^{\beta+1-\alpha} \\
& +\xi_{i}(\beta+1-\alpha)\left(\eta_{i}-s\right)^{\alpha-\beta-1}(1-s)^{\beta-\alpha} \\
= & \sum_{s \leq \eta_{i}} \xi_{i}\left(\eta_{i}-s\right)^{\alpha-\beta-2}(1-s)^{\beta-\alpha}\left[(\alpha-\beta-1)(1-s)+(\beta+1-\alpha)\left(\eta_{i}-s\right)\right] \\
= & \sum_{s \leq \eta_{i}} \xi_{i}\left(\eta_{i}-s\right)^{\alpha-\beta-2}(1-s)^{\beta-\alpha}(\alpha-\beta-1)\left(1-\eta_{i}\right) \geq 0 .
\end{aligned}
$$

Then we have $p$ is a nondecreasing function on $[0,1]$, which implies that $p(s) \geq p(0)=$ $\sum_{i=1}^{\infty} \xi_{i} \eta_{i}^{\alpha-\beta-1}>0, s \in[0,1]$.

Lemma 4 The function $G(t, s)$ defined by (3) admits the following properties:

(i) $G(t, s)>0, \frac{\partial}{\partial t} G(t, s)>0,0<t, s<1$;

(ii) $\max _{t \in[0,1]} G(t, s)=G(1, s)=\frac{1}{p(0) \Gamma(\alpha)}\left[p(s)(1-s)^{\alpha-\beta-1}-p(0)(1-s)^{\alpha-1}\right], 0 \leq s \leq 1$;

(iii) $G(t, s) \geq t^{\alpha-1} G(1, s), 0 \leq t, s \leq 1$.

Proof (i) For $0<s \leq t<1$, noticing that $2<\alpha \leq 3$, by Lemma 3, is easy to see that

$$
\begin{aligned}
G(t, s) & =\frac{1}{p(0) \Gamma(\alpha)}\left[p(s)(1-s)^{\alpha-\beta-1} t^{\alpha-1}-p(0)(t-s)^{\alpha-1}\right] \\
& \geq \frac{1}{p(0) \Gamma(\alpha)}\left[p(0)(1-s)^{\alpha-1} t^{\alpha-1}-p(0)(t-s)^{\alpha-1}\right] \\
& =\frac{1}{\Gamma(\alpha)} t^{\alpha-1}\left[(1-s)^{\alpha-1}-\left(1-\frac{s}{t}\right)^{\alpha-1}\right] \geq 0 .
\end{aligned}
$$

It is clear that for $0<t \leq s<1, G(t, s)>0$.

By direct computation, we have

$$
\frac{\partial}{\partial t} G(t, s)=\frac{1}{p(0) \Gamma(\alpha)}\left\{\begin{array}{rr}
(\alpha-1) p(s)(1-s)^{\alpha-\beta-1} t^{\alpha-2} & \\
-(\alpha-1) p(0)(t-s)^{\alpha-2}, & 0 \leq s \leq t \leq 1 \\
(\alpha-1) p(s)(1-s)^{\alpha-\beta-1} t^{\alpha-2}, & 0 \leq t \leq s \leq 1 .
\end{array}\right.
$$


It is clear that $\frac{\partial}{\partial t} G(t, s)$ is continuous on $[0,1] \times[0,1]$. For $0<s \leq t<1$, noticing that $1 \leq$ $\beta \leq 2$, we get

$$
\begin{aligned}
\frac{\partial}{\partial t} G(t, s) & =\frac{1}{p(0) \Gamma(\alpha)}\left[(\alpha-1) p(s)(1-s)^{\alpha-\beta-1} t^{\alpha-2}-(\alpha-1) p(0)(t-s)^{\alpha-2}\right] \\
& =\frac{(\alpha-1)}{p(0) \Gamma(\alpha)} t^{\alpha-2}\left[p(s)(1-s)^{\alpha-\beta-1}-p(0)\left(1-\frac{s}{t}\right)^{\alpha-2}\right] \\
& \geq \frac{(\alpha-1) p(s)}{p(0) \Gamma(\alpha)} t^{\alpha-2}\left[(1-s)^{\alpha-2}-\left(1-\frac{s}{t}\right)^{\alpha-2}\right] \geq 0 .
\end{aligned}
$$

It is clear that for $0<t \leq s<1, \frac{\partial}{\partial t} G(t, s)>0$.

(ii) By (i), we know that $G(t, s)$ is increasing with respect to $t$, thus we have $\max _{t \in[0,1]} G(t$, $s)=G(1, s)=\frac{1}{p(0) \Gamma(\alpha)}\left[p(s)(1-s)^{\alpha-\beta-1}-p(0)(1-s)^{\alpha-1}\right], 0 \leq s \leq 1$.

(iii) For $0 \leq s \leq t \leq 1$, we get

$$
\begin{aligned}
G(t, s) & =\frac{1}{p(0) \Gamma(\alpha)}\left[t^{\alpha-1} p(s)(1-s)^{\alpha-\beta-1}-p(0)(t-s)^{\alpha-1}\right] \\
& =\frac{1}{p(0) \Gamma(\alpha)} t^{\alpha-1}\left[p(s)(1-s)^{\alpha-\beta-1}-p(0)\left(1-\frac{s}{t}\right)^{\alpha-1}\right] \\
& \geq \frac{1}{p(0) \Gamma(\alpha)} t^{\alpha-1}\left[p(s)(1-s)^{\alpha-\beta-1}-p(0)(1-s)^{\alpha-1}\right] \\
& =t^{\alpha-1} G(1, s) .
\end{aligned}
$$

For $0 \leq t \leq s \leq 1$, we have

$$
\begin{aligned}
G(t, s) & =\frac{1}{p(0) \Gamma(\alpha)} t^{\alpha-1} p(s)(1-s)^{\alpha-\beta-1} \\
& =t^{\alpha-1} \cdot \frac{1}{p(0) \Gamma(\alpha)} p(s)(1-s)^{\alpha-\beta-1} \\
& \geq t^{\alpha-1} G(1, s) .
\end{aligned}
$$

We make the following assumptions:

$\left(\mathrm{H}_{1}\right) f:\left(J \times \mathbb{R}_{++}, \mathbb{R}_{+}\right)$is continuous.

$\left(\mathrm{H}_{2}\right)$ There exist $a, b \in C\left(J, \mathbb{R}_{+}\right), g \in C\left(\mathbb{R}_{++}, \mathbb{R}_{+}\right)$such that

$$
f(t, u) \leq a(t) g(u)+b(t), \quad \forall t \in J, u \in \mathbb{R}_{++}
$$

and

$$
a_{r}^{*}=\int_{0}^{1} a(t) g_{r}(t) \mathrm{d} t<+\infty
$$

for any $r>0$, where

$$
g_{r}(t)=\max \left\{g(u): t^{\alpha-1} r \leq u \leq r\right\}
$$


and

$$
b^{*}=\int_{0}^{1} b(t) \mathrm{d} t<+\infty
$$

$\left(\mathrm{H}_{3}\right)$ There exists $c \in C\left(J, \mathbb{R}_{+}\right)$such that

$$
\frac{f(t, u)}{c(t) u} \rightarrow+\infty \quad \text { as } u \rightarrow+\infty
$$

uniformly for $t \in J$, and

$$
c^{*}=\int_{0}^{1} c(t) \mathrm{d} t<+\infty
$$

$\left(\mathrm{H}_{4}\right)$ There exists $d \in C\left(J, \mathbb{R}_{+}\right)$such that

$$
\frac{f(t, u)}{d(t)} \rightarrow+\infty \quad \text { as } u \rightarrow 0^{+}
$$

uniformly for $t \in J$, and

$$
d^{*}=\int_{0}^{1} d(t) \mathrm{d} t<+\infty
$$

Let $E=C[0,1]$ be the Banach space equipped with the maximum norm $\|u\|=$ $\max _{0 \leq t \leq 1}|u(t)|$ and let $P$ be the cone of nonnegative functions in $C[0,1]$ with the following form:

$$
P=\left\{u \in E \mid u(t) \geq t^{\alpha-1}\|u\|, t \in[0,1]\right\} .
$$

Denote $P_{+}=\{u \in P:\|u\|>0\}$ and $P_{m n}=\{u \in P, m \leq\|u\| \leq n\}$ for any $n>m>0$.

Define the operator $T$ as follows:

$$
(T u)(t)=\int_{0}^{1} G(t, s) f(s, u(s)) \mathrm{d} s, \quad 0 \leq t \leq 1 .
$$

Clearly, $T: P \backslash\{0\} \rightarrow C[0,1]$.

Lemma 5 Suppose that $\left(\mathrm{H}_{1}\right)$ and $\left(\mathrm{H}_{2}\right)$ hold, then for any $j>i>0, T: P_{i j} \rightarrow P$ is completely continuous.

Proof For any $u \in P_{i j}$, then $i \leq\|u\| \leq j$. By the definition of cone $P$, we have

$$
i t^{\alpha-1} \leq u(t) \leq j, \quad \forall t \in[0,1] .
$$

It is not difficult to see that condition $\left(\mathrm{H}_{2}\right)$ implies that

$$
a_{i j}^{*}=\int_{0}^{1} a(t) g_{i j}(t) \mathrm{d} t<+\infty
$$


for any $j>i>0$, where

$$
g_{i j}(t)=\max \left\{g(u): i t^{\alpha-1} \leq u \leq j\right\} .
$$

By $\left(\mathrm{H}_{1}\right),\left(\mathrm{H}_{2}\right)$, (3), and Lemma 4(ii), we get

$$
f(t, u(t)) \leq a(t) g_{i j}(t)+b(t), \quad \forall t \in J
$$

and

$$
\begin{aligned}
(T u)(t) & =\int_{0}^{1} G(t, s) f(s, u(s)) \mathrm{d} s \\
& \leq \frac{1}{p(0) \Gamma(\alpha)} \int_{0}^{1}\left[p(s)(1-s)^{\alpha-\beta-1}-p(0)(1-s)^{\alpha-1}\right] f(s, u(s)) \mathrm{d} s \\
& \leq \frac{1}{p(0) \Gamma(\alpha)} \int_{0}^{1} p(s)(1-s)^{\alpha-\beta-1} f(s, u(s)) \mathrm{d} s \\
& \leq \frac{1}{p(0) \Gamma(\alpha)} \int_{0}^{1}\left[a(s) g_{i j}(s)+b(s)\right] \mathrm{d} s \\
& \leq \frac{1}{p(0) \Gamma(\alpha)}\left(a_{i j}^{*}+b^{*}\right), \quad \forall t \in[0,1],
\end{aligned}
$$

which implies that $T$ is well defined. By Lemma 4(iii), we have

$$
\begin{aligned}
(T u)(t) & =\int_{0}^{1} G(t, s) f(s, u(s)) \mathrm{d} s \\
& \leq \int_{0}^{1} G(1, s) f(s, u(s)) \mathrm{d} s, \quad \forall t \in[0,1]
\end{aligned}
$$

and

$$
\begin{aligned}
(T u)(t) & =\int_{0}^{1} G(t, s) f(s, u(s)) \mathrm{d} s \\
& \geq t^{\alpha-1} \int_{0}^{1} G(1, s) f(s, u(s)) \mathrm{d} s, \quad \forall t \in[0,1]
\end{aligned}
$$

which means that $T$ maps $P_{i j}$ into $P$.

Next, we are in a position to show that $T$ is completely continuous. Let $u_{n}, \bar{u} \in P_{i j}, \| u_{n}-$ $\bar{u} \| \rightarrow 0(n \rightarrow \infty)$, then $\lim _{n \rightarrow \infty} u_{n}(t)=\bar{u}(t), t \in[0,1]$. Let

$$
\begin{aligned}
& \left(T_{1} u\right)(t)=f(t, u(t)), \quad 0<t<1, u \in P_{i j} \\
& \left(T_{2} u\right)(t)=\int_{0}^{1} G(t, s) u(s) \mathrm{d} s, \quad 0<t<1, u \in L^{1}[0,1] .
\end{aligned}
$$

By $\left(\mathrm{H}_{1}\right)$,

$$
\lim _{n \rightarrow \infty} f\left(t, u_{n}(t)\right)=f(t, \bar{u}(t)), \quad 0<t<1
$$


Similar to (6) and (9), for $u_{n}, \bar{u} \in P_{i j}$, we have

$$
f\left(t, u_{n}(t)\right) \leq a(t) g_{i j}(t)+b(t), \quad f(t, \bar{u}(t)) \leq a(t) g_{i j}(t)+b(t), \quad \forall t \in J
$$

Thus, we have

$$
\left|f\left(t, u_{n}(t)\right)-f(t, \bar{u}(t))\right| \leq 2\left[a(t) g_{i j}(t)+b(t)\right]=\sigma(t) \in L^{1}[0,1]
$$

It follows from (13), (14), and the Lebesgue dominated convergence theorem that $\lim _{n \rightarrow \infty} \int_{0}^{1}\left|\left(T_{1} u_{n}\right)(t)-\left(T_{1} \bar{u}(t)\right)\right| \mathrm{d} t=0$, which implies that $T_{1}: P_{i j} \rightarrow L^{1}[0,1]$ is continuous. By the Arzela-Ascoli theorem, we know that $T_{2}: L^{1}[0,1] \rightarrow C[0,1]$ is completely continuous. As a consequence, $T=T_{2} \circ T_{1}: P_{i j} \rightarrow C[0,1]$ is completely continuous.

In order to prove the main theorem, we state the following Guo-Krasnoselskii fixed point theorem.

Lemma 6 ([29]) Let $\Omega_{1}$ and $\Omega_{2}$ be two bounded open sets in Banach space E such that $\theta \in$ $\Omega_{1}$ and $\bar{\Omega}_{1} \subset \Omega_{2}, A: P \cap\left(\bar{\Omega}_{2} \backslash \Omega_{1}\right) \rightarrow P$ a completely continuous operator, where $\theta$ denotes the zero element of $E$ and $P$ a cone of $E$. Suppose that one of the two conditions holds:

(i) $\|A u\| \leq\|u\|, \forall u \in P \cap \partial \Omega_{1} ;\|A u\| \geq\|u\|, \forall u \in P \cap \partial \Omega_{2}$;

(ii) $\|A u\| \geq\|u\|, \forall u \in P \cap \partial \Omega_{1} ;\|A u\| \leq\|u\|, \forall u \in P \cap \partial \Omega_{2}$.

Then $A$ has a fixed point in $P \cap\left(\bar{\Omega}_{2} \backslash \Omega_{1}\right)$.

\section{Main result}

Theorem 1 Let conditions $\left(\mathrm{H}_{1}\right)-\left(\mathrm{H}_{4}\right)$ be satisfied. Assume in addition that there exists $r>0$ such that

$$
\frac{1}{p(0) \Gamma(\alpha)}\left(a_{r}^{*}+b^{*}\right)<r
$$

where $a_{r}^{*}$ and $b^{*}$ are defined in condition $\left(\mathrm{H}_{2}\right)$. Then the boundary value problem (1) has at least two positive solutions $u^{*}$ and $u^{* *}$ with $0<\left\|u^{*}\right\|<r<\left\|u^{* *}\right\|$.

Proof By Lemma 5, the operator $T$ defined by (5) is completely continuous from $P_{m n}$ into $P$ for any $n>m>0$. We need only to prove that $T$ has two fixed points $u^{*}$ and $u^{* *} \in P_{+}$ with $0<\left\|u^{*}\right\|<r<\left\|u^{* *}\right\|$.

By condition $\left(\mathrm{H}_{3}\right)$, there exists $r_{1}>0$ such that

$$
f(t, u) \geq 2^{\alpha-1}\left(\int_{\frac{1}{2}}^{1} G\left(\frac{1}{2}, s\right) c(s) \mathrm{d} s\right)^{-1} c(t) u, \quad \forall t \in J, u \geq r_{1} .
$$

Choose

$$
r_{2}>\max \left\{2^{\alpha-1} r_{1}, r\right\}
$$

For $u \in P,\|u\|=r_{2}$, we have, by the construction of cone $P$,

$$
u(t) \geq t^{\alpha-1} r_{2} \geq\left(\frac{1}{2}\right)^{\alpha-1} r_{2}>r_{1}, \quad \forall t \in\left[\frac{1}{2}, 1\right] .
$$


So, we get from (16) and (18)

$$
\begin{aligned}
(T u)\left(\frac{1}{2}\right) & =\int_{0}^{1} G\left(\frac{1}{2}, s\right) f(s, u(s)) \mathrm{d} s \\
& \geq 2^{\alpha-1}\left(\int_{\frac{1}{2}}^{1} G\left(\frac{1}{2}, s\right) c(s) \mathrm{d} s\right)^{-1} \int_{\frac{1}{2}}^{1} G\left(\frac{1}{2}, s\right) c(s) u(s) \mathrm{d} s \\
& \geq 2^{\alpha-1}\left(\int_{\frac{1}{2}}^{1} G\left(\frac{1}{2}, s\right) c(s) \mathrm{d} s\right)^{-1} \int_{\frac{1}{2}}^{1} G\left(\frac{1}{2}, s\right) c(s) \mathrm{d} s \cdot\left(\frac{1}{2}\right)^{\alpha-1} r_{2}=r_{2} .
\end{aligned}
$$

Therefore,

$$
\|T u\|=\max _{t \in[0,1]}\|(T u)(t)\| \geq\left\|(T u)\left(\frac{1}{2}\right)\right\| \geq r_{2}=\|u\|, \quad \forall u \in P,\|u\|=r_{2} .
$$

By condition $\left(\mathrm{H}_{4}\right)$, there exists $r_{3}>0$ such that

$$
f(t, u) \geq\left(\int_{\frac{1}{2}}^{1} G\left(\frac{1}{2}, s\right) d(s) \mathrm{d} s\right)^{-1} d(t) r, \quad \forall t \in J, 0<u<r_{3} .
$$

\section{Choose}

$$
0<r_{4}<\min \left\{r_{3}, r\right\}
$$

For $u \in P,\|u\|=r_{4}$, we have

$$
r_{3}>r_{4}=\|u\| \geq r_{4} t^{\alpha-1}>0, \quad \forall t \in J
$$

So, we get

$$
\begin{aligned}
(T u)\left(\frac{1}{2}\right) & =\int_{0}^{1} G\left(\frac{1}{2}, s\right) f(s, u(s)) \mathrm{d} s \\
& \geq\left(\int_{\frac{1}{2}}^{1} G\left(\frac{1}{2}, s\right) d(s) \mathrm{d} s\right)^{-1} \int_{\frac{1}{2}}^{1} G\left(\frac{1}{2}, s\right) d(s) r \mathrm{~d} s \\
& \geq\left(\int_{\frac{1}{2}}^{1} G\left(\frac{1}{2}, s\right) d(s) \mathrm{d} s\right)^{-1} \int_{\frac{1}{2}}^{1} G\left(\frac{1}{2}, s\right) d(s) \mathrm{d} s \cdot r=r>r_{4} .
\end{aligned}
$$

Therefore,

$$
\|T u\|=\max _{t \in[0,1]}\|(T u)(t)\| \geq\left\|(T u)\left(\frac{1}{2}\right)\right\|>r_{4}=\|u\|, \quad \forall u \in P,\|u\|=r_{4} .
$$

On the other hand, for $u \in P,\|u\|=r$, similar to (10), by $\left(\mathrm{H}_{2}\right)$, Lemma 3(iii) and (15), we get

$$
\begin{aligned}
(T u)(t) & =\int_{0}^{1} G(t, s) f(s, u(s)) \mathrm{d} s \\
& \leq \frac{1}{p(0) \Gamma(\alpha)} \int_{0}^{1}\left[p(s)(1-s)^{\alpha-\beta-1}-p(0)(1-s)^{\alpha-1}\right] f(s, u(s)) \mathrm{d} s
\end{aligned}
$$




$$
\begin{aligned}
& \leq \frac{1}{p(0) \Gamma(\alpha)} \int_{0}^{1} p(s)(1-s)^{\alpha-\beta-1} f(s, u(s)) \mathrm{d} s \\
& \leq \frac{1}{p(0) \Gamma(\alpha)} \int_{0}^{1}(1-s)^{\alpha-\beta-1} f(s, u(s)) \mathrm{d} s \\
& \leq \frac{1}{p(0) \Gamma(\alpha)}\left(a_{r}^{*}+b^{*}\right)<r, \quad \forall t \in[0,1] .
\end{aligned}
$$

Thus, from (25), we get

$$
\|T u\|<\|u\|, \quad \forall u \in P,\|u\|=r .
$$

We know from (20), (24), (26), and Lemma 6 that $T$ has two fixed points $u^{*}, u^{* *} \in P_{r_{4} r_{2}}$ such that $0<r_{4}<\left\|u^{*}\right\|<r<\left\|u^{* *}\right\| \leq r_{2}$.

\section{An example}

Example 1 Consider the following infinite-point boundary value problems:

$$
\left\{\begin{array}{l}
D_{0+}^{\frac{5}{2}} u(t)+\frac{1}{15 \sqrt[9]{(1-t)}}\left(u^{2}+\frac{1}{6 \sqrt{u}}\right)+\frac{1}{20 \sqrt[3]{t(1-t)}}=0, \quad 0<t<1, \\
u(0)=0, \quad D_{0+}^{\frac{5}{4}} u(0)=0, \quad D_{0+}^{\frac{5}{4}} u(1)=\sum_{i=1}^{\infty} \frac{3}{7} i^{-2} D_{0+}^{\frac{5}{4}} u\left(\frac{1}{i}\right) .
\end{array}\right.
$$

Conclusion BVP (27) has at least two positive solutions $u^{*}$ and $u^{* *}$ with $0<\left\|u^{*}\right\|<r<$ $\left\|u^{* *}\right\|$.

Proof In this problem, $\alpha=\frac{5}{2}, \beta=\frac{5}{4}, \xi_{i}=\frac{3}{7} i^{-2}, \eta_{i}=\frac{1}{i}, f(t, u)=\frac{1}{20 \sqrt[8]{(1-t)}}\left(u^{2}+\frac{1}{3 \sqrt{u}}\right)+\frac{1}{15 \sqrt[3]{t(1-t)}}$. By simple computation, we have $\Gamma\left(\frac{5}{2}\right)=1.3293, \sum_{i=1}^{\infty} \xi_{i} \eta_{i}^{\alpha-\beta-1}=0.6258<1, p(0)=0.3742$. For any $r>0$, it is easy to see that $\left(\mathrm{H}_{2}\right)$ holds for $a(t)=\frac{1}{15 \sqrt[9]{(1-t)}}, g(u)=u^{2}+\frac{1}{6 \sqrt{u}}, b(t)=$ $\frac{1}{20 \sqrt[3]{t(1-t)}}$, and

$$
a_{r}^{*}=\int_{0}^{1} a(t) g_{r}(t) \mathrm{d} t<\int_{0}^{1} \frac{1}{15 \sqrt[9]{(1-t)}}\left(r^{2}+\frac{1}{6 t^{\frac{3}{4}} \sqrt{r}}\right) \mathrm{d} t<+\infty,
$$

$b^{*}=\int_{0}^{1} \frac{1}{20 \sqrt[3]{t(1-t)}} \mathrm{d} t=0.1027$. Obviously, $\left(\mathrm{H}_{3}\right)$ and $\left(\mathrm{H}_{4}\right)$ hold for $c(t)=d(t)=\frac{1}{15 \sqrt[9]{(1-t)}}$, and $c^{*}=d^{*}=0.0600$. Take $r=1$, we have, by (27),

$$
\begin{aligned}
\frac{1}{p(0) \Gamma(\alpha)}\left(a_{1}^{*}+b^{*}\right) & <\frac{1}{0.3742 \times 1.3293} \times(0.0600+0.0463+0.1027) \\
& =0.4202<1=r .
\end{aligned}
$$

Consequently, (15) holds, and our conclusion follows from Theorem 1.

\section{Competing interests}

The authors declare that they have no competing interests.

\section{Authors' contributions}

The authors, XZ and QZ, contributed to each part of this work equally and read and approved the final version of the manuscript.

\section{Author details}

'Department of Information Engineering, Jining Medical College, Jining, Shandong 272067, P.R. China. ${ }^{2}$ School of Mathematics, Liaocheng University, Liaocheng, Shandong 252059, P.R. China. 


\section{Acknowledgements}

The project is supported financially by the Natural Science Foundation of Shandong Province of China (ZR2015AL002), a Project of Shandong Province Higher Educational Science and Technology Program (J15LI16), the Foundations for Jining Medical College Natural Science (JYQ14KJ06, JY2015KJ019, JY2015BS07), and the National Natural Science Foundation of China $(11571197,11571296,11371221,11071141)$

Received: 24 January 2016 Accepted: 7 March 2016 Published online: 21 March 2016

\section{References}

1. Bai, Z: On positive solutions of a nonlocal fractional boundary value problem. Nonlinear Anal. 72, 916-924 (2010)

2. Li, C, Luo, X, Zhou, Y: Existence of positive solutions of the boundary value problem for nonlinear fractional differential equations. Comput. Math. Appl. 59, 1363-1375 (2010)

3. Xu, X, Fei, X: The positive properties of Green's function for three point boundary value problems of nonlinear fractional differential equations and its applications. Commun. Nonlinear Sci. Numer. Simul. 17, 1555-1565 (2012)

4. Xu, X, Jiang, D, Hu, W, O'Regan, D, Agarwal, RP: Positive properties of Green's function for three-point boundary value problems of nonlinear fractional differential equations and its applications. Appl. Anal. 91, 323-343 (2012)

5. Lv, Z: Positive solutions of $m$-point boundary value problems for fractional differential equations. Adv. Differ. Equ. 2011, Article ID 571804 (2011)

6. Yang, J, Ma, J, Zhao, S, Ge, Y: Fractional multi-point boundary value problem of fractional differential equations. Math. Pract. Theory 41(11), 188-194 (2011)

7. Li, B, Sun, S, Li, Y, Zhao, P: Multi-point boundary value problems for a class of Riemann-Liouville fractional differential equations. Adv. Differ. Equ. 2014, 151 (2014)

8. Li, S, Zhang, X, Wu, Y, Caccetta, L: Extremal solutions for $p$-Laplacian differential systems via iterative computation. Appl. Math. Lett. 26, 1151-1158 (2013)

9. Peng, L, Zhou, Y: Bifurcation from interval and positive solutions of the three-point boundary value problem for fractional differential equations. Appl. Math. Comput. 257, 458-466 (2015)

10. Zhang, $X, L i u, L, W u, Y$ : The uniqueness of positive solution for a singular fractional differential system involving derivatives. Commun. Nonlinear Sci. Numer. Simul. 18, 1400-1409 (2013)

11. Wang, $L$, Zhang, X: Positive solutions of $m$-point boundary value problems for a class of nonlinear fractional differential equations. J. Appl. Math. Comput. 42, 387-399 (2013)

12. Zhang, $X$ : Positive solutions for a class of singular fractional differential equation with infinite-point boundary value conditions. Appl. Math. Lett. 39, 22-27 (2015)

13. Rehman, M, Khan, R: Existence and uniqueness of solutions for multi-point boundary value problems for fractional differential equations. Appl. Math. Lett. 23, 1038-1044 (2010)

14. Zhang, $X, L i u, L, W u, Y, L u, Y$ : The iterative solutions of nonlinear fractional differential equations. Appl. Math. Comput. 219, 4680-4691 (2013)

15. Zhang, $X, L i u, L, W u, Y$ : The uniqueness of positive solution for a fractional order model of turbulent flow in a porous medium. Appl. Math. Lett. 37, 26-33 (2014)

16. Zhang, X, Liu, L, Wiwatanapataphee, $B$, Wu, Y: The eigenvalue for a class of singular $p$-Laplacian fractional differential equations involving the Riemann-Stieltjes integral boundary condition. Appl. Math. Comput. 235, 412-422 (2014)

17. Ahmad, B, Ntouyas, SK: Existence results for a coupled system of Caputo type sequential fractional differential equations with nonlocal integral boundary conditions. Appl. Math. Comput. 266, 615-622 (2015)

18. Vong, SK: Positive solutions of singular fractional differential equations with integral boundary conditions. Math. Comput. Model. 57, 1053-1059 (2013)

19. Henderson, J, Luca, R: Positive solutions for a system of fractional differential equations with coupled integral boundary conditions. Appl. Math. Comput. 249, 182-197 (2014)

20. Yuan, C: Two positive solutions for $(n-1,1)$-type semipositone integral boundary value problems for coupled systems of nonlinear fractional differential equations. Commun. Nonlinear Sci. Numer. Simul. 17, 930-942 (2012)

21. Wang, Y, Liu, L, Wu, Y: Positive solutions for a nonlocal fractional differential equation. Nonlinear Anal. 74, 3599-3605 (2011)

22. Cabada, A, Wang, G: Positive solutions of nonlinear fractional differential equations with integral boundary value conditions. J. Math. Anal. Appl. 389, 403-411 (2012)

23. Ahmad, B, Agarwal, RP, Alsaedi, A: Fractional differential equations and inclusions with semiperiodic and three-point boundary conditions. Bound. Value Probl. 2016, 28 (2016)

24. Li, B, Sun, S, Han, Z: Successively iterative method for a class of high-order fractional differential equations with multi-point boundary value conditions on half-line. Bound. Value Probl. 2016, 5 (2016)

25. Zhang, H: Iterative solutions for fractional nonlocal boundary value problems involving integral conditions. Bound. Value Probl. 2016, 3 (2016)

26. Samko, SG, Kilbas, AA, Marichev, OI: Fractional Integrals and Derivatives: Theory and Applications. Gordon \& Breach, Yverdon (1993)

27. Podlubny, I: Fractional Differential Equations. Mathematics in Science and Engineering, vol. 198. Academic Press, New York (1999)

28. Kilbas, AA, Srivastava, HM, Trujillo, JJ: Theory and Applications of Fractional Differential Equations. North-Holland Mathematics Studies, vol. 204. Elsevier, Amsterdam (2006)

29. Guo, D, Lakshmikantham, V: Nonlinear Problems in Abstract Cones. Academic Press, San Diego (1988) 